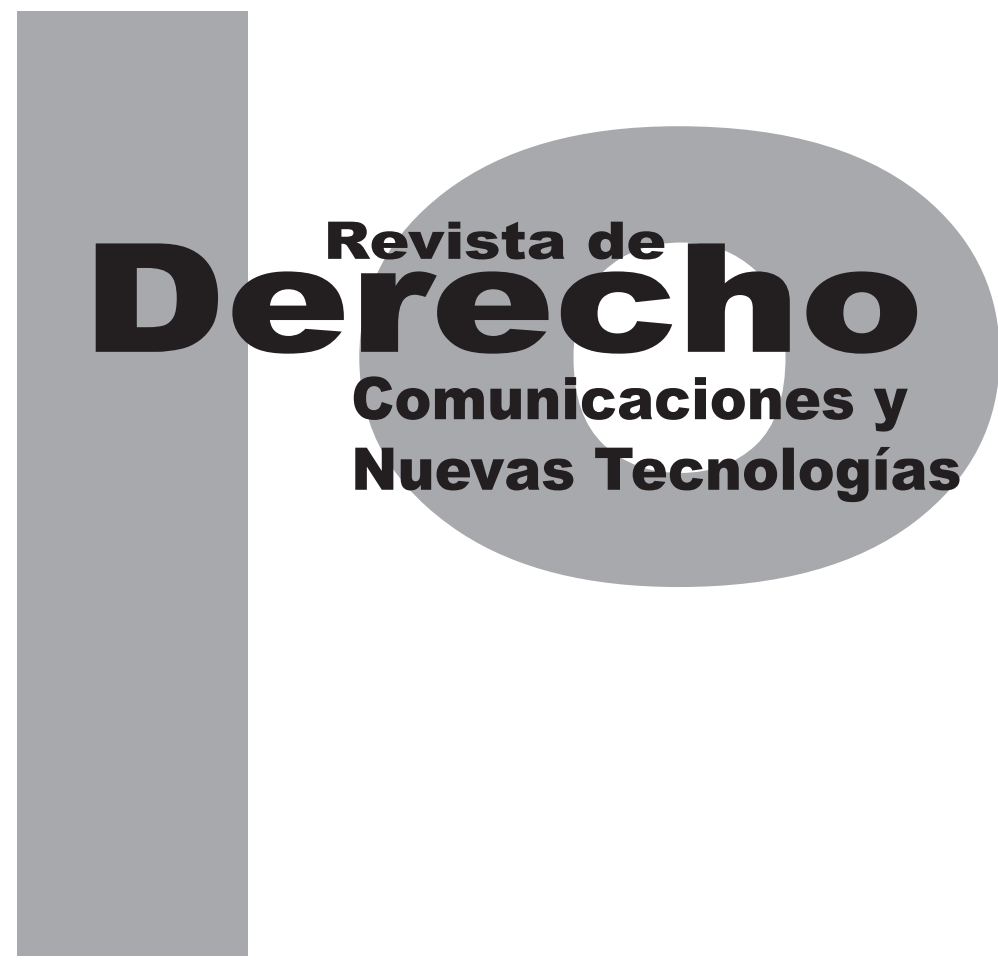

\title{
LoS PAgARÉS ELECTRÓNICOS en el DeReChO ESTADOUNIDENSE
}

\author{
Mariliana Rico CARRILlo
}

Universidad de los Andes

Facultad de Derecho

Revista de Derecho, comunicaciones y Nuevas Tecnologías

N. ${ }^{\circ}$, Junio de 2012. ISSN 1909-7786 


\title{
Los pagarés electrónicos en el Derecho estadounidense $^{1}$
}

\author{
Mariliana Rico Carrillo²
}

\section{RESUMEN}

En Estados Unidos de América, los pagarés electrónicos son objeto de regulación bajo la noción “archivos transferibles", consagrada tanto en el ámbito del derecho de los estados como a nivel federal. Parte de esta normativa fue elaborada con la finalidad de facilitar la emisión y circulación electrónica de estos títulos en el sector inmobiliario y agilizar el otorgamiento de hipotecas, ofreciendo la alternativa de realizar todo el proceso de manera electrónica. En el presente trabajo nos ocupamos del estudio de estos títulos cambiarios, con particular énfasis en los requisitos que permiten el tratamiento de estos archivos electrónicos en igualdad de condiciones a los tradicionales títulos emitidos en papel.
In the United States of America, the electronic promissory notes are subject to regulation under the definition of "transferable records" established in both, state law and federal law. Part of this regulation was developed in order to facilitate the issuance and electronic circulation of these negotiable instruments in real estate, and to facilitate the granting of mortgages, offering the alternative of making the entire process electronically. In this paper we address the study of these negotiable instruments, with particular emphasis on the requirements that allow the processing of electronic files in conditions equal to those of traditional documents issued in paper.

El presente trabajo ha sido realizado en el marco del proyecto de investigación "Títulos valores electrónicos: en particular, títulos cambiarios electrónicos" (DER 2009-11959), financiado por la Dirección General de Investigación del Ministerio de Educación y Ciencia de España.

2 Doctora en Derecho mención Cum Laude por la Universidad Carlos III de Madrid, España (2003). Profesora titular de Derecho Mercantil y Derecho de las Tecnologías de la Información y Comunicación en la Universidad Católica del Táchira (Venezuela). Profesora visitante (categoría: Titular) en el Departamento de Derecho Privado de la Universidad Carlos III de Madrid (2009). Profesora invitada en el máster en Derecho Privado y Doctorado en Derecho de la Universidad Carlos III de Madrid. Profesora invitada en programas de máster y especialización en la Universidad Complutense de Madrid, Universidad de Chile y Universidad de los Andes de Venezuela. Profesora de Derecho de las Nuevas Tecnologías en el Doctorado en Ciencias, mención Derecho de la Universidad Central de Venezuela. Árbitro especializado en Nuevas Tecnologías, certificado por la Corte Suprema de Florida, de los Estados Unidos de América. mariliana@ricocarrillo.com 
Palabras clave: Títulos cambiarios electró- Keywords: Electronic negotiable instrunicos. Pagaré electrónico. Firma electrónica. ments. Electronic promissory notes. Electronic Medios electrónicos de pago. Signature. 


\section{SUMARIO}

Introducción - I. ADMISIBILIDAD DE LOS TÍTULOS CAMBIARIOS EMITIDOS EN SOPORTES ELECTRÓNICOS - A. El concepto de instrumentos negociables en las normas del Uniform Commercial Code - B. La emisión de instrumentos negociables electrónicos - 1. El principio de equivalencia funcional y su aplicación al ámbito de los instrumentos negociables - 2. La regulación de los transferable records - II. EL TRATAMIENTO JURÍDICO DEL PAGARÉ ELECTRÓNICO - A. Condiciones de emisión - 1. La configuración del transferable record como pagaré - 1.1. El cumplimiento de los requisitos de forma - 1.2. La inserción de la firma electrónica - 1.3. La voluntad del emisor - 1.4. La exigencia de un ejemplar único - B. Características básicas del ejemplar único - 1. La identificación de la persona que posee el control del archivo - 2. La inalteración del documento - C. Reglas que rigen la transmisión - 1. La importancia del derecho de control - 2. Sistemas que acreditan el derecho de control: la intervención de terceras partes - D. La posición del deudor y del acreedor del pagaré electrónico - III. EL TRATAMIENTO ELECTRÓNICO DE OTROS TÍTULOS CAMBIARIOS - 1. El procesamiento electrónico de cheques - 2. La implementación de mecanismos electrónicos en el pago mediante cheques - IV. CONCLUSIONES - Bibliografía. 


\section{Introducción}

Desde hace algunos años el fenómeno de la desmaterialización de los títulos-valores se ha hecho presente, sobre todo en el campo de los títulos de participación y tradición. Las anotaciones contables y el tratamiento electrónico de los conocimientos de embarque constituyen el punto de partida del denominado proceso de "electronificación" de los títulos-valores.

La evolución y el desarrollo del comercio electrónico han puesto de manifiesto la necesidad de completar este proceso mediante su expansión a los títulos cambiarios. Las transacciones que se desarrollan en este ámbito exigen la modificación del soporte documental en que tradicionalmente se han representado estos títulos para adecuarlos al entorno negocial electrónico. Esta tarea no es fácil, toda vez que debe venir acompañada de una regulación específica que garantice las condiciones de seguridad que ofrece la emisión en papel.

Pocas legislaciones en el mundo se han ocupado de estudiar el proceso de electronificación de los títulos cambiarios. Las normas de mayor trascendencia en este ámbito han sido desarrolladas en el Derecho estadounidense, en el marco de la Uniform Electronic Transaction Act y la Electronic Signatures in Global and National Commerce Act, consideradas como una regulación sin precedentes en la evolución de los títulos-valores ${ }^{3}$ que surge precisamente en respuesta al desarrollo del comercio electrónico.
Aunque estas normas contemplan la posibilidad de emisión y circulación de los títulos cambiarios en soportes electrónicos, su aplicación se restringe a la figura del pagaré; sobre este aspecto es importante destacar que parte de esta legislación fue aprobada con la finalidad de establecer el régimen jurídico aplicable a estos títulos cambiarios electrónicos y agilizar el otorgamiento de hipotecas, de allí que hayamos escogido este singular título como objeto de nuestro estudio. Dentro de esta normativa también se contempla la posibilidad del procesamiento electrónico de cheques, aunque a partir de un instrumento emitido en papel. En la presente investigación nos ocupamos del estudio de estas cuestiones, en particular de las condiciones que rodean la emisión y circulación de los pagarés electrónicos en el marco de la legislación estadounidense.

\section{ADMISIBILIDAD DE LOS TÍTULOS CAMBIARIOS EMITIDOS EN SOPORTES ELECTRÓNICOS}

Antes de establecer las condiciones de admisibilidad de los títulos cambiarios en soporte electrónico, consideramos pertinente realizar una breve revisión de la legislación general sobre títulos-valores en Estados Unidos de América (EUA), exploración que nos ayudará a lograr una mejor comprensión del proceso de electronificación experimentado en este ámbito.

3 Vid. ILLESCAS ORTIZ, Rafael. Derecho de la contratación electrónica. Civitas, Madrid, 2009, p. 332. 


\section{A. El concepto de instrumentos negociables en las normas del Uniform Commercial Code}

En EUA, los títulos cambiarios, conformados tradicionalmente por las letras de cambio, cheques y pagarés, en su concepción de instrumentos negociables, son regulados en el Uniform Commercial Code (en adelante UCC), elaborado por la National Conference of Commissioners on Uniform State Laws (NCCUSL) ${ }^{4}$ con la finalidad de introducir en el ámbito del Derecho estadounidense un conjunto de reglas de contenido uniforme aplicable a las transacciones comerciales.

En el marco del UCC, los títulos cambiarios son objeto de regulación en el artículo 3 (104), bajo la categoría de instrumentos negociables (negotiable instruments). De acuerdo con esta norma, un instrumento negociable es un documento que contiene una promesa o una orden de pagar una cantidad determinada de dinero. En esta amplia definición se incluyen las letras de cambio y los cheques en su consideración de órdenes de pago, y el pagaré, cuya naturaleza jurídica se identifica con una promesa de pago.

De acuerdo con el artículo 3 (103) del UCC, una promesa de pago es un compromiso por escrito firmado por la persona que asume la obligación de pagar una determinada cantidad de dinero.

4 La NCCUSL, conocida también como la Uniform Law Commision, es una organización compuesta por representantes de los diferentes estados de EUA, que se encarga de debatir sobre las áreas en que debe haber uniformidad en las diferentes legislaciones estatales. La organización fue creada en 1892 con el propósito de superar las dificultades legales que surgen debido a las grandes variantes que se presentan entre las leyes estatales. En el desarrollo de este objetivo, la NCCUSL se encarga de elaborar las leyes uniformes con el objeto de que sean adoptadas en los diferentes estados. Vid. http://www.nccusl. org (Consulta: 03 de diciembre de 2011).
En cuanto a las órdenes de pago, la citada norma indica que se trata de una instrucción por escrito de pagar una cantidad de dinero, firmada por la persona que emite la correspondiente instrucción. Esta orden de pago puede ser dirigida a cualquier persona, incluyendo la propia persona que emite la orden de pago ${ }^{5}$.

En las definiciones citadas resalta la exigencia del documento escrito y de la firma de quien suscribe la promesa o la orden de pago, como requisitos necesarios para emitir el instrumento. En relación con la firma, el artículo 3 (401) del UCC indica que ésta puede ser insertada en el documento manualmente o por medio de un dispositivo o máquina, a través del uso de cualquier nombre, palabra, marca o símbolo adoptados por una persona con la intención de autenticar el documento escrito. Al igual que sucede en el Derecho continental, las normas del UCC también exigen la firma en el endoso del documento, como un requisito necesario para transmitir el derecho incorporado en el título.

\section{B. La emisión de instrumentos negociables electrónicos}

\section{El principio de equivalencia funcional y su aplicación al ámbito de los instrumentos negociables}

El principio de equivalencia funcional, propio del Derecho del comercio electrónico ${ }^{6}$, es con-

5 En este caso, la orden de pago se transforma en una promesa de pago, en el entendido que es el mismo emisor quien se compromete a pagar la cantidad de dinero especificada en el instrumento de pago.

6 Vid. ILLESCAS ORTIZ, Rafael. "La equivalencia funcional como principio elemental del Derecho del comercio electrónico", en Derecho y Tecnología, Universidad Católica del Táchira, No. 1, 2002, pp. 9-24. 
sagrado por primera vez en el artículo 5 de la Ley Modelo de la CNUDMI sobre Comercio Electrónico ${ }^{7}$ (en adelante LMCCE). De acuerdo con los postulados de esta norma: "No se negarán efectos jurídicos, validez o fuerza obligatoria a la información por la sola razón de que esté en forma de mensaje de datos." El principio enunciado tiene por objeto reconocer valor y otorgar eficacia jurídica a los documentos electrónicos, independientemente del soporte material, del procedimiento utilizado en su elaboración y del tipo de firma incorporada. En relación con la firma electrónica, el principio de equivalencia funcional, primariamente consagrado en el artículo 7 de la LMCCE, es incorporado también en el artículo 6 de la Ley Modelo de la CNUDMI sobre Firmas Electrónicas ${ }^{8}$.

En la actualidad, la mayoría de los países han aprobado normas encaminadas a otorgar eficacia jurídica a los mensajes de datos y a las firmas electrónicas, introduciendo en sus respectivas legislaciones el principio de equivalencia funcional, como máxima elemental del Derecho del comercio electrónico. EUA no ha sido la excepción; en el ámbito del Derecho estadounidense, el principio de equivalencia funcional se encuentra consagrado en el marco de la Uniform Electronic Transactions Act (UETA), aprobada por la NCCUSL en 1999 y en la Electronic Signature Global (E-Sing), promulgada como ley federal en el año 2001.

$7 \quad$ NACIONES UNIDAS. Ley Modelo de la CNUDMI sobre Comercio Electrónico con la Guía para su incorporación al derecho interno 1996. Naciones Unidas, Nueva York, 1997.

8 NACIONES UNIDAS. Ley Modelo de la CNUDMI sobre Firmas Electrónicas con la Guía para su incorporación al derecho interno 2001. Naciones Unidas, Nueva York, 2001.
En el marco de la UETA, el principio de equivalencia funcional se encuentra incluido en la Sección 7, al indicar que no se negarán efectos jurídicos a los mensajes de datos o a las firmas electrónicas por el simple hecho de estar representados en formato electrónico. En el ámbito de E-Sing, la Sección 101 (a) reconoce la aplicación del principio de equivalencia funcional al señalar que no se negarán efectos jurídicos a las firmas, contratos o cualquier otra información por el sólo hecho de estar en formato electrónico. De acuerdo con estas normas, cuando las leyes exijan como condición de validez el documento escrito o la firma, estos requisitos pueden satisfacerse a través de un documento electrónico o una firma electrónica.

Si bien el principio de equivalencia funcional es aplicable al ámbito de los instrumentos negociables, en el entendido que tanto el documento como la firma exigida para su emisión pueden sustituirse por sus análogos electrónicos, las especiales características de estos instrumentos y su vinculación con el tradicional soporte papel, han exigido una regulación especial, en aras a la adaptación de ciertos conceptos elaborados sobre la base del tradicional documento cartáceo ${ }^{9}$. Esta situación se traduce en una excepción a la aplicación automática de este

9 Tal es el caso de la búsqueda del equivalente funcional de la posesión, como requisito esencial para ejercer el derecho tradicionalmente incorporado al papel. Esta circunstancia ha sido puesta de manifiesto por la doctrina española. En palabras de ALBA FERNÁNDEZ: "El reto que la traslación del fenómeno de la negociabilidad de los documentos en el entorno electrónico vino a plantear en primer término, se identificó entonces con la reproducción o la búsqueda del equivalente funcional de la posesión". Vid. ALBA FERNÁNDEZ, Manuel. "Documentos de transporte y negociabilidad en un entorno electrónico", Revista de Derecho Mercantil, Núm. 263, enero-marzo de 2007, p. 104. 
principio ${ }^{10}$, pues no basta que el documento se represente en forma electrónica y se suscriba también de esta manera; el principio de equivalencia funcional en este ámbito debe venir acompañado de una regulación específica que otorgue las condiciones de garantía y seguridad que ofrecen los tradicionales títulos-valores emitidos en papel ${ }^{11}$.

El problema que se presenta con la aplicación del principio de equivalencia funcional en el campo de los títulos-valores, no se centra en la inserción de la firma electrónica y en el cumplimiento de las formalidades exigidas para la emisión de estos documentos, sino en las especiales características de estos instrumentos, derivadas de su particular vinculación con el tradicional soporte papel, en atención a la exigencia de un ejemplar único y a la necesidad de presentar el documento original para el ejercicio

10 El caso de los títulos-valores constituye una de las excepciones a la aplicación del principio de equivalencia funcional contempladas en las normas del Derecho Uniforme del Comercio Internacional (DUCl). Sobre este aspecto destacan en particular las disposiciones de la Convención de las Naciones Unidas sobre la Utilización de las Comunicaciones Electrónicas en los Contratos Internacionales de 2005, que excluye de su ámbito de aplicación a las letras de cambio y a los pagarés. Esta exclusión no es un impedimento para que los Estados puedan establecer en su legislación las condiciones necesarias para regular el tratamiento electrónico de estos títulos-valores, tal como ha ocurrido con la normativa estadounidense, que fue aprobada con anterioridad a esta Convención.

11 En España, la jurisprudencia ha considerado esta situación. En esta oportunidad cabe mencionar la sentencia de la Audiencia Provincial de Barcelona 326/2010, de 13 de julio, donde se valora la validez de un supuesto pagaré autenticado con firma electrónica reconocida del firmante; en este caso el juzgador indica que el cumplimiento del requisito de firma no es suficiente para caracterizar al documento como un pagaré electrónico, en el entendido que en España «la legislación no regula los pagarés electrónicos, la legalidad de la firma electrónica no cambia nada y el documento electrónico no adquirirá la condición de título-valor que se pretende, por el mero hecho de la legalidad de la firma.» El texto de esta sentencia está disponible en http://portaljuridico. lexnova.es/jurisprudencia/JURIDICO/78118/sentencia-ap-barcelona326-2010-de-13-de-julio-pagare-requisitos-necesidad-de-incorporarel-de (Consulta: 15 de enero de 2012). del derecho incorporado ${ }^{12}$. La intangibilidad del documento y la relevancia jurídica de la presentación del documento original en los títulos emitidos en papel representan un desafío en el entorno electrónico, ante la facilidad de copia de los archivos electrónicos y las dificultades que se presentan a la hora de diferenciar entre un original y una copia. El Derecho estadounidense, a través de la UETA y la E-SIGN ofrece un conjunto de reglas para solucionar los inconvenientes que se derivan de esta situación.

\section{La regulación de los transferable records}

En la normativa estadounidense, los instrumentos negociables electrónicos son objeto de regulación en la UETA y en la E-Sing, bajo la denominación "transferable records". A diferencia de la E-Sing, que es una ley federal, la UETA es una ley uniforme, cuyo texto ha de ser adoptado por los diferentes estados que forman parte de la unión ${ }^{13}$. Ambos instrumentos fueron aprobados con la finalidad de reducir los obstáculos relacionados con el uso de medios electrónicos en el ambiente comercial y regular las transacciones electrónicas, otorgando valor a los documentos y a las firmas electrónicas. La diferencia

12 Respecto a esta condición se ha pronunciado la doctrina española al indicar que «el problema de los documentos cambiarios en soporte electrónico, prescindiendo del soporte papel tradicional, es el problema de la unicidad, por la posibilidad (y enorme facilidad) de copia de los documentos electrónicos, sin posibilidad, además, de distinguir el "original" de sus posteriores "copias"». Vid. MARTíNEZ NADAL, Apollonia. "La admisibilidad jurídica del pagaré electrónico". Diario La Ley, $n^{\circ}$. 7461, año XXXI, septiembre de 2010.

13 En la actualidad, 47 estados de los 50 que forman parte de la unión, junto con Puerto Rico y las Islas Vírgenes han adoptado las normas de la UETA. Vid http://www.ncsl.org/default.aspx?tabid=13484 (Consulta: 12 de noviembre de 2010). 
fundamental entre estas dos normas radica en su ámbito de aplicación: la finalidad de la UETA se centra principalmente en regular el uso de la firma electrónica en el campo empresarial, mientras que la E-Sign incorpora un mayor número de normas encaminadas a garantizar la protección de los consumidores ${ }^{14}$.

La importancia de estos instrumentos jurídicos en el sector de los títulos-valores radica precisamente en la introducción de una serie de normas encaminadas a regular la emisión y transmisión de los instrumentos negociables. Las reglas de la UETA y la E-Sign, junto con la aplicación del principio de equivalencia funcional, facilitan la emisión y transmisión de los instrumentos negociables al permitir la sustitución del tradicional documento escrito por un mensaje de datos signado en forma electrónica.

Los transferable records no son más que el equivalente electrónico de los instrumentos negociables regulados en el UCC. El término "transferable record", traducido al español como archivo electrónico transferible $(A E T)^{15}$ fue utilizado por primera vez en la UETA en 1999, siendo posteriormente incorporado en la E-Sign en el año 2001. La regulación sobre los AET fue introducida en estas normas con la finalidad de reducir el uso de pagarés emitidos en papel en el mercado inmobiliario y agilizar el otorgamiento de hipotecas, ofreciendo la alternativa de realizar todo el proceso de manera electrónica ${ }^{16}$.

14 Vid. Stephanie Lillie. "Will ESIGN force States to adopt UETA?" 42 Jurimetrics J. 21-30 (2001).

15 ILLESCAS ORTIZ, Rafael. Derecho de la contratación electrónica. Civitas, Madrid, 2009, p. 337.

16 WINN, Jane. "What is a Transferable Record and Who Cares? Journal of Science and Technology Laws, Vol. 7: 203, Boston University, 2001 p. 205.
En la actualidad, numerosos prestamistas hipotecarios están utilizando los e-Notes (pagarés electrónicos) ${ }^{17}$, gracias a las normas de la UETA y de la E-Sign, y a las directrices incluidas en las guías para la emisión de pagarés con firmas electrónicas, elaboradas por The Mortgage Industry Standars Maintenance Organization $(\mathrm{MISMO})^{18}$.

De acuerdo con lo establecido en la Sección 16 de la UETA, la noción de AET se refiere a un archivo electrónico que puede ser un pagaré (en los términos del artículo 3 del UCC), un certificado de depósito, un conocimiento de embarque u otro título de tradición (en los términos del artículo 7 del UCC) ${ }^{19}$. Según esta disposición, para que el tradicional título-valor soportado en papel pueda ser sustituido por un AET, se deben cumplir necesariamente dos requisitos: a) que se trate de un pagaré o de un título de tradición, y b) que el emisor del documento haya convenido en forma expresa la utilización del AET.

17 Las estadísticas sobre el uso de pagarés electrónicos del sector hipotecario en los Estados Unidos de América pueden consultarse en: http:// www.mortgagebankers.org/eMortgageDashboard.htm (Consulta: 12 de noviembre de 2010).

18 http://www.mismo.org/specs/emortgage-specs.html (Consulta: 12 de noviembre de 2010).

19 El contenido textual de la norma es el siguiente: In this section, "transferable record" means an electronic record that: (1) would be a note under [Article 3 of the Uniform Commercial Code] or a document under [Article 7 of the Uniform Commercial Code] if the electronic record were in writing; and (2) the issuer of the electronic record expressly has agreed is a transferable record". (Subrayado nuestro). Es de destacar que en el marco del UCC, la definición de "note" se restringe a las promesas de pago, que en el Derecho continental podrían ser bien un pagaré o una letra de cambio girada contra el mismo librador. En este concepto estarían excluidos los cheques y las letras de cambio giradas como órdenes de pago. Así se desprende del contenido textual del artículo 3-104 (e), cuyo tenor es el siguiente: "An instrument is a $\square$ "note" if it is a promise and is a $\square$ "draft" if it is an order." 
En el marco de la E-Sign, la regulación de los AET se encuentra contenida en la Sección 201. En esta norma, los AET son definidos en términos similares a los especificados en la UETA, aunque la regulación se limita únicamente a los pagarés que sean emitidos para garantizar préstamos sobre bienes inmuebles. En este caso, son tres los requisitos que deben cumplirse para la generación del AET: a) que se trate de un pagaré, b) que el emisor del documento haya convenido en forma expresa la utilización del AET, y c) que el pagaré se haya emitido para garantizar un préstamo sobre un bien inmueble. Las previsiones de esta sección contemplan en forma expresa el uso de la firma electrónica en Ios $\mathrm{AET}^{20}$.

Una comparación entre las normas incluidas en la UETA y en la E-Sign en relación con los AET, nos lleva a la conclusión que la definición de los AET contemplada en la UETA es más amplia que la de la E-Sign, ya que abarca no sólo los pagarés electrónicos -independientemente de la causa de su emisión - sino también los títulos de tradición. En relación con la delimitación de la noción $\mathrm{AET}$, debemos apuntar que el comentario oficial a la UETA realizado por la NCCUSL, indica en forma expresa que la definición se circunscribe sólo a los pagarés y a los títulos de tradición, por lo tanto no se aplica a los cheques y a las letras de cambio. Esta limitación encuentra su fundamento en el impacto que puede causar en los grandes sistemas de pago,

20 El contenido textual de la norma es el siguiente: The term "transferable record" means an electronic record that: $(A)$ would be a note under Article 3 of the Uniform Commercial Code if the electronic record were in writing; $(B)$ the issuer of the electronic record has expressly agreed is a transferable record; and (C) relates to a loan secured by real property. A transferable record may be executed using an electronic signature". la electronificación de estos títulos de crédito, en particular del cheque ${ }^{21}$.

\section{EL TRATAMIENTO JURÍDICO DEL PAGARÉ ELECTRÓNICO}

Dentro de los instrumentos negociables electrónicos objeto de esta regulación especial, la figura del pagaré adquiere especial importancia en el entendido que la normativa sobre los transferable records fue elaborada precisamente con la finalidad de facilitar la emisión de pagarés electrónicos en el sector inmobiliario y agilizar el otorgamiento de hipotecas. El crecimiento de las operaciones y el coste que representa el almacenamiento y el manejo de los documentos instrumentados en papel, particularmente cuando éstos se emiten en grandes cantidades -como sucede en el sector hipotecario- junto con el desarrollo de las operaciones comerciales

21 El contenido textual incluido en el comentario número 2, en relación con esta exclusión es del tenor siguiente: "The definition of transferable record is limited in two significant ways. First, only the equivalent of paper promissory notes and paper documents of title can be created as transferable records. Notes and Documents of Title do not impact the broad systems that relate to the broader payments mechanisms related, for example, to checks. Impacting the check collection system by allowing for "electronic checks" has ramifications well beyond the ability of this Act to address. Accordingly, this Act excludes from its scope transactions governed by UCC Articles 3 and 4." En relación con esta limitación, la doctrina española acertadamente indica que el régimen de los AET no es aplicable a las letras de cambio y a los cheques (que son los documentos regulados en los artículos 3 y 4 del UCC), y que el comentario a los artículos 3 (b) (2) y 16 (a) (1) justifica la limitada aplicación a los pagarés y a los títulos de tradición “... en la necesidad de no afectar a los sistemas de pago y sustentar la temporal exclusión en los efectos o riesgos sistémicos y en la falta de una tecnología que asegure la singularidad y control de estos instrumentos". Vid. MORILLAS JARILLO, María José. "Letra de cambio electrónica", Revista de la Contratación Electrónica, No. 31, 2002, p. 40. En relación con esta excepción, la doctrina anglosajona considera que: "Without any experience to predict the risks of loss and hence no adequate basis for developing new regulations, banks regulators balked at the notion of authorizing electronic checks. As a result, the transferable record provisions of both UETA and E-Sign do not extend to checks." Vid. WINN, Jane. "What is a Transferable Record and Who Cares? Óp. cit., p. 207. 
electrónicas, originaron la necesidad de elaborar un conjunto de reglas especialmente destinadas a regular estos aspectos, con la finalidad de establecer las condiciones que debe cumplir un documento electrónico para desempeñar la función equivalente a la de un pagaré emitido en papel.

En relación con los otros títulos de crédito (cheques y letras de cambio), el ámbito de aplicación de esta normativa es limitado, en el entendido que la definición de los transferable records, referida a los títulos de crédito, se circunscribe únicamente al pagaré, en su consideración de promesa de pago. No obstante esta limitación, consideramos necesario mencionar que en el caso del cheque, tanto la UETA como la E-SIGN, establecen que cuando la ley exija la retención de un cheque, este requisito puede ser satisfecho a través de la retención del archivo electrónico que contiene la información del instrumento de pago, esto implica que el tratamiento electrónico del cheque solo es posible a partir de un título-valor emitido en papel. La sustitución del cheque en papel por un soporte electrónico ha sido objeto de regulación en una ley federal, de la que nos ocupamos en el último apartado de esta investigación.

Aunque en el caso de los títulos de crédito tanto la UETA como la E-SIGN limitan esta noción al pagaré electrónico ${ }^{22}$, en el marco de la E-SIGN el

22 La E-SIGN únicamente permite la emisión de pagarés electrónicos bajo la figura de los transferable records, circunstancia que se deduce de la definición incluida en la Sección 201, al indicar que el término transferable record "... means an electronic record that- $(A)$ would be a note under Article 3 of the Uniform Commercial Code if the electronic record were in writing." En cuanto a la admisión de los títulos-valores en soporte electrónico, la UETA es más amplia, ya que también permite la emisión de los títulos incluidos en el artículo 7 del UCC (certificados de depósito y conocimientos de embarque electrónico). Bajo concepto es aún más restringido, ya que se refiere únicamente a los pagarés electrónicos que sean emitidos para garantizar préstamos sobre bienes inmuebles. Esta circunstancia obedece a la finalidad primaria de esta ley, orientada a garantizar la protección de los derechos de los consumidores en las transacciones electrónicas, donde este tipo de pagaré adquiere especial importancia ${ }^{23}$. En atención a estas consideraciones centramos nuestro estudio en las previsiones de la E-SIGN, sin dejar de mencionar las disposiciones de la UETA cuando sea necesario.

\section{A. Condiciones de emisión}

De acuerdo con las reglas incluidas en la Sección 201 de la E-SIGN, son tres los requisitos que deben cumplir los AET para que puedan considerarse como equivalentes funcionales de los pagarés emitidos en papel. En primer lugar, que se trate de un pagaré, esto es de un documento que cumpla las condiciones legalmente exigidas para que pueda considerarse como tal; en segundo lugar, que el emisor haya convenido en forma expresa la utilización del AET como medio para documentar su obligación y, por último, que el pagaré se emita para garantizar un préstamo sobre un bien inmueble. El cumplimiento de estos requisitos es básico para que pueda

\footnotetext{
Ios postulados de la UETA, un transferable record "would be a note under [Article 3 of the Uniform Commercial Code] or a document under [Article 7 of the Uniform Commercial Code] if the electronic record were in writing."

23 En palabras de la doctrina estadounidense: "The limitation of the scope of the E-Sign transferable record provision to notes security by real property was due in part to concerns of consumer advocates that too broad a transferable record provisions might permit unscrupulous merchants and finance companies to receive old scams in new electronic forms..." Vid. WINN, Jane. "What is a Transferable Record and Who Cares? Óp. cit.
} 
aplicarse el principio de equivalencia funcional al archivo electrónico en toda su extensión.

\section{La configuración del transferable record como pagaré electrónico}

\subsection{Cumplimiento de los requisitos de forma}

En el caso de los pagarés electrónicos, aun cuando la representación del título-valor se hace a través de un soporte inmaterial, el derecho se mantiene incorporado en el documento electrónico representativo del título. En razón de esta circunstancia, el archivo electrónico debe reunir las mismas características de su homólogo en papel.

Para que el AET sea catalogado como un títulovalor, en particular como un pagaré (promissory note o simplemente "note" en terminología anglosajona), el documento debe cumplir las condiciones exigidas en la Sección 3-104 del UCC; a estos requisitos se adiciona la firma de quien se compromete al pago (el emisor del pagaré).

En el Derecho estadounidense el pagaré puede ser un instrumento negociable o no negociable. Para que sea considerado un documento negociable, las normas del UCC exigen el cumplimiento de las siguientes condiciones: a) emisión en papel, b) firma del emisor, c) inclusión de la promesa incondicional de pagar una cantidad específica de dinero, c) determinación del momento de pago (que puede ser a la vista o en un plazo determinado), y d) ser pagadero a la orden de una determinada persona o de la persona que esté en posesión del título.
En la configuración del pagaré electrónico deben cumplirse las especificaciones indicadas en la ley para la emisión de los pagarés en papel, este es el primer requisito exigido en las normas en estudio, no hay que olvidar que la especial particularidad en este caso reside en la naturaleza intangible del soporte donde se encuentra incorporado el derecho. Independientemente de la tecnología utilizada en la elaboración del documento, el archivo electrónico para que sea tratado jurídicamente como un pagaré debe incluir la promesa incondicional de pago, el momento de pago y la persona facultada para exigir el pago, a menos que se trate de un instrumento emitido al portador ${ }^{24}$. En este caso, la exigencia del papel como soporte documental se sustituye por el archivo electrónico, igual que sucede con la firma manuscrita, que es sustituida por la firma electrónica. Seguidamente pasamos al análisis del cumplimiento de este requisito en el entorno electrónico.

\subsection{La inserción de la firma electrónica}

Al tratarse de un documento de naturaleza intangible, el requisito de firma sólo puede cumplirse a través de la inserción de la firma electrónica en el documento. En el ámbito de los AET, las disposiciones de la E-SIGN y de la UETA permiten en forma expresa la utilización de la firma electrónica en la generación del archivo, aunque no

24 En el caso de los pagarés electrónicos en el sector inmobiliario The Mortgage Industry Standars Maintenance Organization (MISMO) ha elaborado un conjunto de directrices que permiten la emisión de pagarés electrónicos de conformidad con las previsiones de la E-SIGN. La tecnología empleada en este caso se basa principalmente en la aplicación del lenguaje XML que permite la inclusión de las menciones legales necesarias para considerar el AET como un equivalente al tradicional pagaré. Vid. http://www.mismo.org/Guidelines (Consulta: 15 de enero de 2011) 
establecen un determinado tipo de firma para que sea procedente aplicar el principio de equivalencia funcional entre la firma electrónica y la firma manuscrita, como sucede en las legislaciones de otros países. Ambos textos se limitan a consagrar el principio de no discriminación del soporte, al indicar que no se negarán efectos jurídicos a las firmas por el sólo hecho de estar en formato electrónico, circunstancia que obedece al respeto del principio de neutralidad tecnológica. En atención a esta máxima, y siguiendo los postulados de las Leyes Modelo de UNCITRAL, la definición de firma electrónica en la E-SIGN -y también en la UETA- se refiere en general, a cualquier tecnología adoptada por una persona con la finalidad de otorgar autoría al documento, en este caso, al documento electrónico.

En los AET, serán las partes quienes pacten el tipo de firma a utilizar, lo importante es que la tecnología escogida permita identificar al firmante, en este caso al emisor del pagaré. En la generación del AET también es fundamental la utilización de un sistema de información fiable, que permita la identificación de la persona que posee el control del documento (quien equivale al tenedor en los títulos emitidos en papel). Es importante recordar que tanto la UETA como la E-SIGN son adoptadas con la finalidad de proporcionar seguridad jurídica a las personas que voluntariamente deciden instrumentar sus relaciones a través de medios electrónicos y someterse a la aplicación de esta normativa ${ }^{25}$, sin imponer una tecnología específica. En aplicación de estos principios, corresponde a las partes

25 ROJAS AMANDI, Víctor. "La Uniform Electronic Transactions Act de los Estados Unidos de América". Boletín Mexicano de Derecho, 119, Universidad Autónoma de México, p. 548. la selección de la tecnología que habrá de emplearse para la inserción de la firma electrónica en el respectivo pagaré, así como la especificación de los términos y condiciones para determinar la validez de esta forma de autenticación electrónica.

\subsection{La voluntad del emisor}

La voluntad del emisor es un presupuesto fundamental para la sustitución del papel por un AET como forma de documentar la obligación subyacente en el respectivo pagaré. Para que el documento electrónico pueda ser considerado un pagaré, es necesaria la aprobación del emisor, cuya manifestación de voluntad se expresa con la inserción de su firma, en este caso de su firma electrónica.

La exigencia de la conformidad del emisor en la generación del archivo asegura que éste no pueda ser obligado a aceptar la conversión de un documento emitido originalmente en papel por un AET sin su expresa aprobación. Esta exigencia es acorde con los principios básicos del comercio electrónico, en particular con la autonomía de la voluntad de las partes, pues ellas mismas son quienes deciden la forma en que van a documentar sus obligaciones. Aunque el acuerdo contractual es fundamental en este ámbito, es importante apuntar que el acuerdo por sí solo no es suficiente para elaborar un título-valor en soporte electrónico con las mismas características de los títulos en papel. Si no existieran leyes como la UETA o la E-SIGN, no sería posible la sustitución del título-valor en papel por un título electrónico que otorgase a los sujetos intervinientes los mismos derechos que 
confiere el tradicional instrumento negociable. El acuerdo por lo tanto tiene por objeto aceptar la aplicación de la normativa propia que rige los AET, es en el marco de este acuerdo donde se determina la naturaleza del título y la tecnología que se va a utilizar en la generación del AET. En nuestro caso de estudio, debe tratarse de un pagaré, y por aplicación de los principios de la E-SIGN, un pagaré emitido para garantizar obligaciones inmobiliarias.

En relación con los aspectos tecnológicos que rodean la emisión del $\mathrm{AET}$, las normas exigen la utilización de un sistema de información seguro o confiable (reliable), que garantice un nivel adecuado de seguridad por lo que respecta a la emisión, conservación y transferencia del archivo. Ni la E-SIGN ni la UETA establecen estándares tecnológicos para la emisión y conservación de los archivos, ninguno de estos textos determina los requisitos que debe cumplir el sistema de información, en el entendido que lo importante en estos casos es el resultado ${ }^{26}$. Son las partes, quienes mediante el respectivo acuerdo contractual, deben seleccionar el sistema de información que cumpla las exigencias legales, de acuerdo con la importancia de sus negocios y la evolución de la tecnología ${ }^{27}$. El resultado exigido según la ley, es la determinación, en todo momento, de la identidad de la persona que posee el control del AET (el titular del derecho incorporado). La selección de la tecnología aplicable queda en manos de las partes, quienes

26 Esta orientación de las leyes estadounidenses encuentra su fundamento en el respeto al principio de neutralidad tecnológica, establecido primariamente en la Ley Modelo de UNCITRAL sobre comercio electrónico.

RAS AMANDI, Víctor. "La Uniform Electronic Transactions Act..., óp. cit., p. 560 escogerán el sistema que cumpla los requisitos exigidos en la ley, atendiendo a la tecnología imperante en el mercado ${ }^{28} \mathrm{y}$ al nivel de seguridad exigido.

\subsection{La existencia de un ejemplar único}

Uno de los mayores problemas que suscita la aplicación del principio de equivalencia funcional en el ámbito de los títulos-valores se refiere a la exigencia de un ejemplar único y a la necesidad de presentar el documento original para el ejercicio del derecho incorporado, elementos que caracterizan la emisión de los tradicionales títulos en papel. Estas dos exigencias son básicas, ya que es en el documento donde finalmente está incorporado el derecho, circunstancia que se mantiene en el caso de los AET, donde el derecho se incorpora a un soporte de naturaleza intangible.

La intangibilidad del documento y la relevancia jurídica de la presentación del documento original en los títulos emitidos en papel representan un desafío en el entorno electrónico, ante la facilidad de copia de los archivos electrónicos y las dificultades que se presentan a la hora de diferenciar entre un original y una copia.

El problema de los originales y copias en el entorno electrónico se deriva principalmente de los requisitos que deben cumplir los mensajes de datos para ser considerados como documentos originales, en el entendido que en el mundo

\footnotetext{
28 NATION, George. "Electronic Contracting after E-Sign: The Impact on Commercial Loan Documentation", Commercial Lending Review, 2001, p. 33.
} 
electrónico, el procesamiento y la transmisión de los documentos implica necesariamente la generación de una copia. En estos casos, para determinar la originalidad se atiende al criterio de la integridad (inalterabilidad del documento), introducido primariamente en el ámbito de la LMCCE $^{29}$.

En atención al criterio de la integridad, habrá tantos originales como documentos inalterados existan. Esta situación plantea toda una problemática en el sector de los títulos-valores por la especial naturaleza de estos documentos y la importancia que representa el derecho incorporado, en el entendido que se trata de un único derecho incorporado en un único título. Estas circunstancias han exigido la revisión de la legislación, con la finalidad de establecer una regulación propia para los documentos electrónicos en su condición de equivalentes a los tradicionales títulos-valores (diversa de la aplicable a los mensajes de datos en general), a efectos de garantizar la existencia de un ejemplar único y evitar la proliferación de fraudes, debido a la facilidad de reproducción que ofrece actualmente la tecnología.

La característica del ejemplar único mencionada en los textos legales exige que el sistema de información empleado permita distinguir el

29 En el texto de esta norma, el criterio de la inalterabilidad del documento electrónico (mensaje de datos) como requisito esencial para satisfacer las condiciones de un documento original se encuentra establecido en el artículo 8 al indicar que "... Cuando la ley requiera que la información sea presentada y conservada en su forma original, ese requisito quedará satisfecho con un mensaje de datos: a) Si existe alguna garantía fidedigna de que se ha conservado la integridad de la información a partir del momento en que se generó por primera vez en su forma definitiva, como mensaje de datos o en alguna otra forma...". De acuerdo con el apartado tercero de esta disposición, la integridad ha de ser evaluada atendiendo al criterio de inalteración de la información. documento original (authoritative copy) de las simples copias. Las normas utilizan la expresión "copia autorizada" en atención al funcionamiento de los archivos electrónicos, cuya transmisión necesariamente implica la reproducción del documento. Una copia se considera autorizada cuando identifica al sujeto que posee el control del archivo, quien es el único facultado para hacer cambios o transmitir el AET, en su condición de titular del derecho incorporado. Para que la copia adquiera la condición de tal, además de tratarse de un documento único, debe ser identificable e inalterable.

\section{B. Características básicas del ejemplar único}

Las características que debe cumplir el ejemplar único para ser considerado como tal, se reconducen a dos: la identificación de la persona que posee el control del documento (controller), que en el caso de los títulos-valores emitidos en papel sería el tenedor (holder) y la inalterabilidad del AET, esta última circunstancia está sometida a un estricto régimen de excepciones que se derivan de la necesidad de alterar el AET cuando hay una transferencia de derechos. Seguidamente pasamos a analizar estas características.

\section{La identificación de la persona que posee el control del archivo}

En el entorno electrónico, el requisito de la posesión -elemento indispensable de los títulosvalores emitidos en papel para ejercer el derecho incorporado- se sustituye por el derecho de 
control. La noción de control se introduce en la legislación estadounidense con la finalidad de establecer un equivalente que permita determinar la persona que está facultada para el ejercicio del derecho, en este caso, ya no se habla de posesión, sino del control del AET, ni de tenedor sino de controlador (controller). Para poder ejercer sus derechos, el "controlador" deberá probar que efectivamente dispone del control del documento electrónico. El estudio del derecho de control es analizado en infra, en esta oportunidad solo queremos poner de manifiesto la importancia de identificar a la persona que posee el control del AET.

La característica de negociabilidad de los AET permite la circulación del archivo junto con la transferencia de derechos de una persona a otra en cualquier momento, esto significa que quien posee el control del AET no siempre será la misma persona. Cualquiera que detente el control, bien sea porque el archivo fue emitido originalmente a su nombre o porque lo adquirió mediante una transferencia, puede transmitir el derecho incorporado a otro sujeto, quien a su vez asumirá la condición de nuevo controlador, con las mismas facultades que el controlador original, de ahí la importancia de acreditar el requisito de identidad en todo momento. En atención a estas circunstancias, el sistema de información designado para la generación y transmisión de Ios AET debe permitir no sólo la identificación del controlador original sino de los sucesivos controladores, de manera que pueda distinguir- se claramente quién es el acreedor del derecho incorporado en un momento determinado ${ }^{30}$.

\section{La inalterabilidad del documento}

La inalterabilidad significa integridad, en otras palabras, que el documento no haya sido modificado. Aunque la regla general para garantizar la condición de documento original es la inalterabilidad, en el ámbito de los AET se admiten ciertas excepciones. El establecimiento de estas excepciones deriva de la naturaleza esencialmente transmisible de los AET, en su condición de instrumentos negociables. El régimen de excepciones encuentra su fundamento en el artículo 8 de la LMUCE, que dispone que la integridad de la información será evaluada conforme al criterio de que haya permanecido completa e inalterada, salvo la adición de algún endoso o de algún cambio que sea inherente al proceso de comunicación, archivo o presentación del mensaje de datos.

En los AET, las excepciones a la regla de la inalterabilidad se reducen a tres situaciones:

a. Cuando hay una transferencia de titularidad. Toda transferencia debe ser consentida por la persona que acredite el control del documento, que es finalmente el legitimado a transmitir el derecho incorporado.

b. Cuando se solicitan copias del ejemplar autorizado. Siempre que se solicite un duplicado el sistema de información debe indicar que se trata de una simple copia.

30 ILLESCAS ORTÍZ, R. Derecho de la contratación electrónica. Óp. cit., p. 336.

I6 Junio de 2012 - Universidad de los Andes - Facultad de Derecho - Revista de Derecho, comunicaciones y nuevas tecnologías. GECTI N. 7 
c. Cuando procede la revisión o modificación del contenido del AET. El sistema debe indicar que se trata de una modificación autorizada por la persona que controla el documento.

\section{Reglas que rigen la transmisión}

\section{La importancia del derecho de control}

La negociación del instrumento no significa otra cosa que la cesión de la posesión mediante su entrega al nuevo tenedor, acto que a su vez implica la transmisión del derecho incorporado.

Al igual que sucede en el Derecho continental, las normas que rigen la transmisión de los instrumentos negociables incluidas en la Sección 3-201 del UCC, exigen la cesión de la posesión y el endoso del título, a menos que se trate de un título al portador (Sección 3-204), cuya transmisión se verificará únicamente con la entrega.

De acuerdo con los principios de equivalencia funcional e inalteración del Derecho preexistente de obligaciones y contratos, para realizar una transmisión electrónica del instrumento negociable deberían cumplirse los mismos requisitos que se exigen en la negociabilidad del instrumento en papel: acreditación de la posesión y endoso del documento, acto que se lleva a cabo mediante la firma y la entrega. Si bien esto pareciera sencillo en un primer momento, veremos que en la práctica no lo es, de ahí que se advierta una excepción en cuanto a la aplicación de estos principios. En el entorno electrónico, el cumplimiento de estos requisitos se sustituye por la noción del "control" del documento, como veremos más adelante.

En los AET, la circulación del instrumento se lleva a cabo a través de redes de comunicación, circunstancia que genera inconvenientes para probar la posesión del documento y acreditar su carácter de original; no hay que olvidar que se trata de documentos de naturaleza intangible, diseñados específicamente para cumplir las funciones de los tradicionales títulos-valores y circular en este entorno. En estos casos, tanto la UETA como la E-SIGN permiten que el requisito de la posesión se acredite mediante el control del documento. La representación electrónica del título-valor y su negociabilidad en un contexto diferente al habitual -intangible por naturaleza- han exigido la reformulación del concepto tradicional de la posesión, vinculado a la tenencia material del papel como condición necesaria para ejercer y transmitir el derecho incorporado en el título. En los AET, el endoso y la entrega del instrumento se sustituyen por la transferencia del control exclusivo, elemento que permite y facilita la circulación del título en el entorno electrónico.

La adquisición del control se construye como un equivalente funcional de la posesión y sustituye a ésta, a la entrega y al endoso de los títulos en papel ${ }^{31}$, de esta manera, la persona que tiene el control del documento -y puede demostrar esta situación- asume la posición de titular legítimo del derecho incorporado al so-

31 MORILLAS JARILLO, María José. "Letras de cambio electrónicas". Óp. cit., p. 38. 
porte electrónico ${ }^{32}$. El derecho de control en los términos indicados en la UETA y en la E-SIGN, ha sido incorporado en diversas secciones del UCC, donde resaltan las disposiciones incluidas en el artículo 9 (Revised Article 9 UCC) ${ }^{33}$. Este conjunto normativo es conocido como "New eCommerce Laws", aunque ya han pasado más de diez años desde su aprobación.

En relación con la atribución del control a una persona determinada, tanto la UETA como la E-SIGN indican que una persona tiene el control del documento electrónico cuando el sistema utilizado establece de manera fiable que esa persona es el sujeto para el cual el AET ha sido emitido o transmitido ${ }^{34}$ y que se trata de un documento original, único e inalterable, salvo las excepciones indicadas anteriormente respecto de este último requisito.

Ambos cuerpos legales establecen una serie de criterios para facilitar la prueba de esta circunstancia, indicando que quien alegue ser el controlador del AET debe proveer una prueba razonable (reasonable proof) que acredite esta

32 En relación con el control del AET y la posesión de los títulos en papel, la doctrina estadounidense al comentar las normas de la UETA indica que no se trata de crear un mecanismo idéntico a la posesión, en el entendido que las leyes destinadas a regular los AET simplemente introducen un conjunto de estándares que producen el mismo resultado de la posesión en los títulos en papel. De ahí que la entrega, la posesión y el endoso sean sustituidos por la noción de control. En este sentido, WHITAKER, David. "Rules Under the Uniform Electronic Transactions Act for an Electronic Equivalent to a Negotiable Promissory Note". Business Law, 55, November, 1999, p. 450.

33 Un estudio sobre las modificaciones de este artículo en el ámbito que nos ocupa puede ser consultado en: Gary A. Goodman. "Revised UCC Article 9: Its Broad and Varied Impact on Real Estate Finance". Disponible en http://www.akingump.com/docs/publication/359.pdf (Consulta: 15 de diciembre de 2011).

34 En términos de la Sección 201, apartado (b) de E-SIGN “... A person has control of a transferable record if a system employed for evidencing the transfer of interests in the transferable record reliably establishes that person as the person to which the transferable record was issued or transferred..." circunstancia. Entre las pruebas mencionadas en los textos legales se incluye el acceso a la copia autorizada para establecer la identidad de la persona que posee el control del documento ${ }^{35}$.

\section{Sistemas que acreditan el derecho de control: la intervención de terceras partes}

Los requisitos para acreditar el control en las condiciones legalmente exigidas pueden ser satisfechos mediante la intervención de un tercero de confianza, encargado de administrar el sistema de información que se utilizará en la gestión, almacenamiento y transmisión del pagaré electrónico.

En la actualidad existen distintos sistemas para acreditar el derecho de control y las condiciones de unicidad, identidad e inalterabilidad de los AET. Recordemos que bajo las previsiones legales para que el AET sea considerado un pagaré, el sistema de información utilizado debe establecer de manera confiable el cumplimiento de estas condiciones (Safe Harbour).

Entre los modelos existentes para satisfacer estas condiciones destacan principalmente dos: el Single Document Managment System (SDMS), que permite a todas las partes el acceso a la copia autorizada en un solo sistema de información (single computer network) ${ }^{36}$ y el Registry Model

35 En el caso de la E-SIGN, la norma se encuentra consagrada en el apartado (f) de la sección 201 y es del tenor siguiente: "Proof may include access to the authoritative copy of the transferable record and related business records sufficient to review the terms of the transferable record and to establish the identity of the person having control of the transferable record."

36 Este sistema ha evolucionado hacia el Multiple Interactive System (MIS) donde cada una de las partes administra su propio sistema de información para proteger la copia autorizada e identificar la persona que posee el control. La transferencia de control mediante 
(RM), donde la copia autorizada es transmitida de un sistema a otro a través de una central de registro. Ambos sistemas están diseñados para cumplir las especificaciones legales y desarrollar las funciones básicas en torno a la acreditación del control y de las condiciones que debe cumplir la copia autorizada, en el sentido que permiten establecer la ubicación de ésta, identificar la persona que detenta el control y realizar de manera efectiva la transferencia del AET.

En el caso del SDMS, la copia autorizada es creada y almacenada en un entorno electrónico seguro denominado E-vault (bóveda electrónica). Cualquier persona que necesite acceder al documento lo hará a través del E-vault ${ }^{37}$. El método de seguridad empleado se basa en la identificación de la parte que detenta el control para cada AET y en la implantación de los controles necesarios para permitir la transferencia e identificar las copias no autorizadas.

El sistema de registro (RM) ofrece la posibilidad de almacenar el AET en una base de datos centralizada que permite el acceso a los interesados y la transferencia del control. Bajo este modelo, el control del AET y la ubicación de la copia autorizada se determinan únicamente por

la utilización de este mecanismo se lleva a cabo a través del envío de la copia autorizada de un sistema a otro. Vid. Mortgage Bankers Association (MBA). "Security Interests in Transferable Records Evidencing Residential Mortgage Lending Transactions and the Rights of Warehouse Lenders", 2007. http://www.mortgagebankers.org/files/ Conferences/2008/2008LIRC/LIRC08GracePowersUsingTechnology. pdf (Consulta: 16 de diciembre de 2011).

37 En el marco de este sistema destaca la iniciativa Transferable Records Management (TRM) de eOriginal, empresa de desarrollo de software que proporciona una plataforma tecnológica basada en el funcionamiento del E-vault para las transacciones que se llevan a cabo mediante la constitución de hipotecas garantizadas con pagarés electrónicos. Vid. http://www.eoriginal.com/solutions/transferable-records-management (Consulta: 28 de diciembre de 2011). la referencia al registro ${ }^{38}$. Las condiciones para el registro exigen que todas las partes interesadas en la creación y transferencia del control del documento acuerden la utilización del registro centralizado para establecer el control y dar seguimiento a la transferencia del título. En este caso, la entidad central (que puede ser pública o privada) $)^{39}$ se encarga de procesar la transmisión del AET. La transferencia se lleva a cabo mediante la recepción de una autorización de quien controla el documento. Este sistema es considerado el más eficaz para dar cumplimiento a las condiciones establecidas en los cuerpos legales que regulan los $\mathrm{AET}^{40}$.

Lo importante en cualquiera de los casos es que el sistema de información designado para la emisión y transmisión del AET pueda establecer de manera confiable la identidad de la persona que posee el control del documento y las condiciones de unicidad, identificación e inalterabilidad (Safe Harbour). Si el sistema no pue-

38 El uso de sistemas de registro a través de bases de datos centralizadas para identificar a la parte que tiene el control del documento está mencionado en el comentario oficial del artículo 16 de la UETA al indicar que "The control requirements may be satisfied through the use of a trusted third party registry system". En cuanto a la eficacia del sistema, la nota del comentarista indica que "A system relying on a third party registry is likely the most effective way to satisfy the requirements of subsection (c) that the transferable record remain unique, identifiable and unalterable, while also providing the means to assure that the transferee is clearly noted and identified" Si bien el comentario oficial se refiere al modelo de registro centralizado, también se admite la utilización de otros sistemas tecnológicos que satisfagan las condiciones legalmente establecidas para que un AET pueda ser tratado como tal.

39 En el sector hipotecario, el modelo de registro es llevado a cabo por Mortgage Electronic Registry Systems (MERS) en la plataforma eRegistry. Los prestamistas e inversionistas registran y transfieren el control de los pagarés electrónicos a través de este sistema Vid. http://www. mersinc.org/membership/WinZip/MERSeRegistryMembershipKit.pdf. (Consulta: 28 de diciembre de 2011).

40 Un estudio comparativo de ambos sistemas es realizado por la American Bar Association. Vid. ABA Cyberspace Committee Working Group on Transferable Records. "Emulating Documentary Tokens in an Electronic Environment: Practical Models for Control and Priority Interests in Transferable Records and Electronic Chattel Paper". Business Law, 59, November, 2003, pp. 385-389. 
de garantizar estas condiciones, se produce un incumplimiento de los requisitos específicamente establecidos para que opere la equivalencia funcional entre el pagaré tradicional y el pagaré electrónico y por lo tanto no puede hablarse de un AET, ni serán de aplicación las disposiciones de la E-SIGN que rigen este tipo de documentos. La evaluación de la seguridad en la tecnología empleada y el cumplimiento de los requisitos legales sólo podrán determinarse a posteriori, una vez obtenidos los resultados de su utilización ${ }^{41}$.

\section{La posición del deudor y del acreedor del pagaré electrónico}

De acuerdo con las previsiones contenidas en la Sección 201 (d) de la E-SIGN, la persona que acredite el control del documento asume la misma posición del tenedor de un instrumento negociable y en consecuencia está facultada para ejercer los derechos incorporados en el soporte electrónico, sin necesidad de cumplir ningún requerimiento adicional. En relación con las defensas del deudor, el apartado (e) de la citada disposición establece que salvo acuerdo en contrario, éste tendrá los mismos derechos y acciones contemplados en las normas del UCC en el caso de los pagarés emitidos en papel. En atención a estas previsiones, el ejercicio de los derechos incorporados en el AET se hará a través de la vía cambiaria.

La introducción de estas normas en la legislación estadounidense no es sino una expresión más de los principios de equivalencia funcional e inalteración del Derecho preexistente de

41 Vid. ILLESCAS ORTÍZ, R. "Derecho de la contratación electrónica". Óp. cit., p. 334. obligaciones y contratos, en el entendido que quien acredita el control del AET asume la misma posición jurídica que el legitimado al cobro en el caso de los títulos emitidos en papel (el tenedor), y quien asume la posición de deudor goza de las mismas defensas que el obligado por un pagaré emitido en papel, salvo que las partes dispongan otra cosa ${ }^{42}$.

Como bien puede observarse, el principio de equivalencia funcional en el campo de los títulos cambiarios electrónicos no sólo se predica entre los documentos electrónicos y los documentos en papel. Las normas también lo establecen respecto de la posición de quien resulta tenedor y legitimado al cobro, esto implica que los derechos y acciones de los tenedores, en cuanto acreedores, son los mismos, independientemente del soporte en que se haya emitido el título ${ }^{43}$, siempre que se cumplan las condiciones legalmente establecidas.

Esta equivalencia entre las posiciones subjetivas de los obligados y titulares de derechos en los pagarés en papel y los sujetos que participan en los AET, se construye con la finalidad de facilitar el ejercicio de los derechos cambiarios a partir de un instrumento negociable electrónico. Es importante insistir que para que la normati-

42 El texto de la norma es del tenor siguiente: "Except as otherwise agreed, a person having control of a transferable record is the holder, as defined in section 1-201(20) of the Uniform Commercial Code, of the transferable record and has the same rights and defenses as a holder of an equivalent record or writing under the Uniform Commercial Code, including, if the applicable statutory requirements under section 3-302(a), 9-308, or revised section 9-330 of the Uniform Commercial Code are satisfied, the rights and defenses of a holder in due course or a purchaser, respectively." La inclusión de este dispositivo reafirma una vez más el poder que asume la voluntad de las partes en el entorno de las negociaciones electrónicas. ILLESCAS ORTIZ, R. "Derecho de la contratación electrónica". Óp. cit., p. 332. 
va específica que rige los AET sea aplicable, el archivo electrónico debe satisfacer los requerimientos legalmente exigidos. En estos casos, la acreditación del control es esencial, en el entendido que la ausencia de este requisito desnaturaliza al archivo electrónico en su consideración de instrumento negociable. Las consecuencias jurídicas que este incumplimiento produce son importantes: si bien el soporte electrónico y la firma del emisor pudieran constituirse como elementos probatorios de la existencia de una obligación, la falta en el cumplimiento de las exigencias legales no permite la configuración del AET como tal, por lo tanto tampoco procede la equivalencia funcional de las posiciones subjetivas ni la aplicación de la normativa cambiaria.

Aunque el tenedor del supuesto AET pudiese probar la existencia de la obligación y reclamar algún derecho, si no logra acreditar el control exclusivo sobre el documento no puede ejercer su derecho valiéndose de las prerrogativas que ofrece la vía cambiaria ${ }^{44}$. Esta circunstancia refleja la importancia del cumplimiento de los requisitos legales, como elemento fundamental para que proceda la equivalencia funcional entre los pagarés electrónicos y los tradicionales pagarés emitidos en papel.

44 Esta situación ha sido puesta de manifiesto por la American Bar Association al indicar que: " $A$ key question in designing a system for administering these types of electronic records is whether the method for registering a transfer of interests reliably establishes the identity of the person entitled to the control of the record. A failure to meet this requirement may be fatal to a claim for status as the equivalent of a holder or purchaser of a Documentary Token. Not that failure to maintain the control does not mean that the underlying obligation can't be enforcement against the obligator-it just means that the transferee may not obtain the special rights that a holder or purchaser of the paper document would get with respect to the defenses and/or any third party claims". Vid. ABA Cyberspace Committee Working Group on Transferable Records. "Emulating Documentary Tokens in an Electronic Enviroment..." Óp. cit., p. 382.

\section{EL TRATAMIENTO ELECTRÓNICO DE OTROS TÍTULOS CAMBIARIOS}

\section{El procesamiento electrónico de cheques}

Tanto la UETA como la E-SIGN permiten el tratamiento electrónico del cheque en el procesamiento del pago, siempre a partir de un instrumento emitido en soporte papel. Ambas normas establecen que cuando la ley exija la retención de un cheque, ese requisito puede ser satisfecho a través de la retención del archivo electrónico que contiene la información del anverso y del reverso del cheque. Este sistema ha sido desarrollado en EUA a través del Check 21.

El Check 21 es un sistema creado en el año 2003, gracias a la aprobación de la Check Clearing for the 21st Century Act (comúnmente conocida como Check 21) con la finalidad de facilitar el procesamiento electrónico de los tradicionales cheques emitidos en papel. Esta regulación introduce la noción del cheque sustituto (Substitute Check), también denominado Image Replace Document (IRD).

El cheque sustituto es un instrumento que contiene la reproducción digital de un cheque originalmente emitido en papel, con la finalidad de facilitar el truncamiento del cheque y agilizar los procedimientos de compensación interbancarios. El IRD tiene las mismas características del cheque emitido en soporte físico, sólo que ha sido digitalizado para facilitar el procesamiento de los pagos. Los cheques sustitutos mantienen su condición de título-valor y son reconocidos 
como tales, siempre que cumplan los requisitos especificados. Estos requisitos se refieren básicamente a la reproducción fiel y exacta del cheque de papel. Para que esta condición se cumpla, los IRD deben contener la imagen del anverso y del reverso del cheque original, el número de cuenta asociado al instrumento de pago, el Bank Routing Number" ${ }^{45}$ y la frase "This is a legal copy of your check. You can use it at the same way you would use the original check" (Esta es una copia legal de su cheque. Se puede utilizar en la misma forma que usaría el cheque original).

El cheque sustituto es creado con la finalidad de facilitar el truncamiento de los cheques, ya que permite el procesamiento y la compensación electrónica del cheque a través de la eliminación del papel y la captura de los datos asociados al instrumento de pago. Los cheques originales son destruidos luego que se han digitalizado y se han creado en su lugar los cheques sustitutos, con las ventajas que esto significa en agilidad y ahorro de costes.

Entre las ventajas que se advierten en el uso del cheque sustituto, encontramos la eliminación del papel en los envíos de los cheques pagados a los libradores del instrumento de pago. En la práctica bancaria estadounidense, los cheques que han sido presentados al cobro son enviados al emisor por correo postal junto con

45 El Bank Routing Number, también conocido como ABA Bank Routing Number, es un número compuesto por nueve dígitos que se encuentra en la parte inferior del cheque y sirve para identificar la institución financiera responsable del pago del cheque. Para facilitar la lectura de este número se utiliza el sistema MICR (Magnetic Ink Character Recognition). MICR es una tecnología de reconocimiento de caracteres utilizada para facilitar la tramitación de los cheques, que permite a los ordenadores leer la información de los documentos impresos. sus estados de cuenta, como prueba del pago. Las previsiones de la Check 21 permiten a los bancos eliminar el envío por correo del cheque emitido originalmente en papel, y sustituirlo por el envío electrónico del cheque sustituto. Es de destacar que sólo los cheques sustitutos que han sido digitalizados por el banco son legalmente equivalentes como prueba de pago. Las fotocopias o las digitalizaciones de los cheques realizadas por los particulares no tienen ningún efecto legal.

\section{La implementación de mecanismos electrónicos en el pago mediante cheques}

Aun cuando la emisión de cheques electrónicos en condiciones similares al pagaré no está regulada en el Derecho estadounidense, recientemente se han implementado mecanismos que permiten el procesamiento electrónico de los pagos realizados mediante el tradicional instrumento cambiario. En este sector destaca el Paper Check Conversion Over the Counter (PCC OTC), conocido en la industria privada como E-Check. Al igual que sucede con el sistema del E-Check 21, el mecanismo utilizado por el PCC OTC exige la emisión del cheque en papel, de ahí que no se pueda hablar de instrumentos totalmente electrónicos.

EI PCC OTC es un sistema que permite la conversión del cheque en una transferencia electrónica de fondos (TEF) mediante la lectura de los datos incluidos en el cheque, que se lleva a cabo a través de un terminal lector instalado en los proveedores de bienes y/o servicios adheridos al sistema. En el momento que se presenta 
el instrumento de pago, el proveedor de bienes y/o servicios aceptante introduce el cheque en el terminal lector, y una vez que la información es captada por el sistema, se utiliza para realizar la transferencia de fondos a favor del aceptante del instrumento de pago. El mecanismo descrito también puede utilizarse para realizar pagos a distancia, en este caso, el emisor envía el cheque por correo, una vez que el título es recibido se procesa la TEF con los datos del instrumento de pago. Jurídicamente, el pago realizado a través de este sistema está gobernado por las leyes sobre TEF. Aunque el pago tiene su origen en un cheque, éste sólo se utiliza como fuente de información para obtener los datos que permitirán realizar la TEF ${ }^{46}$.

\section{CONCLUSIONES}

1. Aun cuando los instrumentos negociables regulados originalmente en el UCC no cumplen las especificaciones requeridas para circular en los entornos electrónicos que caracterizan la nueva economía, las normas del ordenamiento jurídico estadounidense contenidas en la UETA y en la E-Sing introducen las reglas destinadas a regular la emisión de los instrumentos electrónicos equivalentes a los tradicionales títulos de crédito, que originalmente nacieron unidos al papel.

2. Bajo esta regulación se introduce la figura de los transferable records, diseñados para circular específicamente en el ámbito de las

46 Sobre el funcionamiento de este sistema, Vid. https://www.pccotc.gov/ pccotc/pcc/usingpcc/about.htm (Consulta: 30 de noviembre de 2010). operaciones comerciales electrónicas, en los mismos términos que sus análogos en papel y en las condiciones establecidas en la citada normativa. En el estado actual, la emisión de títulos cambiarios electrónicos sólo es posible en el caso del pagaré; así lo establecen en forma expresa los mencionados instrumentos.

3. La configuración de los transferable records como equivalentes a los tradicionales títulos emitidos en papel exige el cumplimiento de determinados requisitos, donde destacan la inserción de la firma electrónica del emisor, el acuerdo contractual y la exigencia de identificar un ejemplar único. Este último requisito se satisface mediante la figura del control sobre el documento electrónico. La falta del cumplimiento de estos requisitos no permite que el documento electrónico adquiera la naturaleza jurídica de un título-valor y por lo tanto impide la aplicación de la normativa cambiaria en las condiciones establecidas en la ley.

4. El derecho de control sobre el archivo electrónico se introduce en la legislación estadounidense con la finalidad de establecer un equivalente de la posesión y sustituye a ésta, a la entrega y al endoso de los títulos en papel. El control se acredita mediante la utilización de un sistema de información seguro que deberá ser suministrado por una tercera parte confiable. El sistema debe identificar, en todo momento, al titular del derecho incorporado.

5. Los sujetos que intervienen en el pagaré electrónico asumen la condición de deudores y 
acreedores con los mismos efectos de las tradicionales posiciones cambiarias, siempre que se cumplan los requisitos establecidos en la legislación especial que regula estos instrumentos. La persona que acredite el control del archivo electrónico asume la misma posición jurídica del tenedor, en tanto que el deudor goza de las mismas defensas que otorga la legislación cambiaria cuando se trata de un título emitido en papel.

6. En el caso de los cheques, aunque su emisión como documentos electrónicos está exceptuada de la normativa en análisis, existen disposiciones que permiten el procesamiento electrónico de estos instrumentos, tal es el caso de la regulación contenida en la Check 21, cuyas normas se aplican a la digitalización del tradicional documento emitido en papel, con la finalidad de facilitar el truncamiento del cheque y agilizar los sistemas de compensación interbancaria. También se han diseñado mecanismos que permiten el procesamiento del pago en forma electrónica, a partir de la emisión de un cheque en papel.

\section{Bibliografía}

ABA Cyberspace Committee Working Group on Transferable Records. "Emulating Documentary Tokens in an Electronic Environment: Practical Models for Control and Priority Interests in Transferable Records and Electronic Chattel Paper". Business Law, 59, November, 2003, pp. 385-389.
ALBA FERNÁNDEZ, Manuel. "Documentos de transporte y negociabilidad en un entorno electrónico". Revista de Derecho Mercantil, Núm. 263, enero-marzo de 2007, pp. 69-113.

ILLESCAS ORTIZ, Rafael. Derecho de la contratación electrónica. Civitas, Madrid, 2009.

ILLESCAS ORTIZ, Rafael. “La equivalencia funcional como principio elemental del Derecho del comercio electrónico". Derecho y Tecnología, Universidad Católica del Táchira, No. 1, 2002, pp. 9-24.

GOODMAN, Gary A. "Revised UCC Article 9: Its Broad and Varied Impact on Real Estate Finance", disponible en http://www.akingump.com/docs/publication/359.pdf (Consulta: 15 de diciembre de 2011).

MARTÍNEZ NADAL, Apollonia. “La admisibilidad jurídica del pagaré electrónico". Diario La Ley, $n^{\circ}$. 7461, año XXXI, septiembre de 2010.

MORILLAS JARILLO, María José. “Letra de cambio electrónica". Revista de la Contratación Electrónica, No. 31, 2002, pp. 17-46.

NATION, George. “Electronic Contracting after E-Sign: The Impact on Commercial Loan Documentation". Commercial Lending Review, 2001.

ROJAS AMANDI, Víctor. "La Uniform Electronic Transactions Act de los Estados Unidos de América". Boletín Mexicano de Derecho, 119, Universidad Autónoma de México, pp. 531-582. 
STEPHANIE, Lillie. "Will ESIGN force States to adopt UETA?" 42 Jurimetrics, 2001, pp. 21-30.

WHITAKER, David. "Rules under the Uniform Electronic Transactions Act for an Electronic Equivalent to a Negotiable Promissory Note". Business Law, 55, November, 1999.
WINN, Jane. "What is a Transferable Record and Who Cares?" Journal of Science and Technology Laws, Boston University, Vol. 7: 203, 2001. 